\title{
TT 33 - Tombe de Padiaménopé (Thèbes-ouest, 2019)
}

Claude Traunecker, Bernard Mathieu, Silvia Einaudi, Isabelle Régen, Gaël Pollin, Olivier Onézime, Simone Nannucci, Faisl Bousta, François Boyer, Philippe Bromblet, Sophie Duberson, Joëlle Le Roux, Anne Liégey, Stéphane Moularat et Stéphanie Touron

\section{(2) OpenEdition} Journals

Édition électronique

URL : https://journals.openedition.org/baefe/1162

DOI : $10.4000 /$ baefe. 1162

ISSN : 2732-687X

Éditeur

ResEFE

\section{Référence électronique}

Claude Traunecker, Isabelle Régen, Silvia Einaudi, Bernard Mathieu, Gaël Pollin, Olivier Onézime, Simone Nannucci, Faisl Bousta, François Boyer, Philippe Bromblet, Sophie Duberson, Joëlle Le Roux, Anne Liégey, Stéphane Moularat et Stéphanie Touron, «TT 33 - Tombe de Padiaménopé (Thèbesouest, 2019) » [notice archéologique], Bulletin archéologique des Écoles françaises à l'étranger [En ligne], Égypte, mis en ligne le 05 octobre 2020, consulté le 29 juillet 2021. URL : http:// journals.openedition.org/baefe/1162 ; DOI : https://doi.org/10.4000/baefe.1162

Ce document a été généré automatiquement le 29 juillet 2021.

Le Bulletin archéologique des Écoles françaises à l'étranger est mise à disposition selon les termes de la Licence Creative Commons Attribution - Pas d'Utilisation Commerciale - Pas de Modification 4.0 International. 


\title{
TT 33 - Tombe de Padiaménopé (Thèbes-ouest, 2019)
}

\author{
Claude Traunecker, Bernard Mathieu, Silvia Einaudi, Isabelle Régen, Gaël \\ Pollin, Olivier Onézime, Simone Nannucci, Faisl Bousta, François Boyer, \\ Philippe Bromblet, Sophie Duberson, Joëlle Le Roux, Anne Liégey, \\ Stéphane Moularat et Stéphanie Touron
}

\section{NOTE DE L'AUTEUR}

Année de la campagne : 2019 (10 octobre - 21 novembre)

Numéro et intitulé de l'opération de terrain : 17146 - La nécropole thébaine au VII siècle av. J.-C. La tombe du prêtre-lecteur Padiamenopé (TT 33, fin XXVe-début XXVI e dynastie) : mission épigraphique et archéologique

Composition de l'équipe de terrain

Mission épigraphique et archéologique :

L'équipe était composée de Claude Traunecker (égyptologue, université de Strasbourg, CNRS, UMR 7044 Archéologie et histoire ancienne : Méditerranée-Europe ArcHiMedE), Silvia Einaudi (égyptologue, CNRS-PSL-EPHE, UMR 8546 Archéologie et philologie d'Orient et d'Occident AOrOc, université de Strasbourg, CNRS, UMR 7044), Isabelle Régen (égyptologue, université Paul-Valéry Montpellier 3, CNRS, UMR 5140 Archéologie des Sociétés Méditerranéennes), Bernard Mathieu (égyptologue, université Paul-Valéry Montpellier 3, CNRS, UMR 5140), Simone Nannucci (archéologue, université de Strasbourg, UMR 7044), Olivier Onézime (topographe-photogrammètre, Ifao), Gaël Pollin, (photographe, Ifao).

Mission d'expertise (assainissement et conservation) :

Faisl Bousta (responsable du pôle Microbiologie, LRMH), François Boyer (pôle conservation préventive, C2RMF), Philippe Bromblet (géologue, CICRP), Sophie Duberson (restauratrice, département des Antiquités égyptiennes, musée du Louvre), Joëlle Le Roux (restauratrice, service de la conservation préventive à la direction de la recherche et des collections du musée du Louvre), Anne Liégey (restauratrice indépendante), Stéphane Moularat (spécialiste des polluants en milieu fermé, Centre 
Scientifique et Technique du bâtiment - CSTB), Stéphanie Touron (responsable du pôle Grottes Ornées, LRMH), Christine Gallois (organisation et coordination, fonds Khéops pour l'archéologie).

Le ministère des Antiquités était représenté par Amal Motasem Mostafa (inspectrice) et Mohammed Hassan (restaurateur).

Partenariats institutionnels : Ce travail bénéficie du soutien du CNRS, UMR 5140, de l'université Paul-Valéry Montpellier 3, du CNRS, UMR 7044, de l'université de Strasbourg et du fonds Khéops pour l'archéologie (Paris).

\section{Problématiques et bilan des principaux résultats}

1 La tombe de Padiamenopé (TT 33) est la plus grande tombe d'Égypte mais sa structure est particulièrement atypique. Si elle a en commun avec les autres grandes tombes de l'Assassif les trois premières salles avec une chapelle de culte, elle possède aussi un ensemble cultuel central (le cénotaphe) avec un couloir de circumambulation. Claude Traunecker a montré que le cénotaphe s'inspire du tombeau d'Osiris à Abydos tel que l'ont restitué des prêtres d'Abydos sous les Ramessides. Les quinze fausses-portes du cénotaphe, ainsi que les trois chapelles XIV, XV et XVI, sont en rapport avec les manipulations et rites de l'embaumement.

2 Le très riche matériel épigraphique de la tombe découle de la volonté explicite de Padiamenopé de mettre à la disposition de visiteurs cultivés tous les grands textes de la littérature funéraire. Isabelle Régen a montré que ces textes ont été repensés et mis en forme par l'érudit qu'était Padiamenopé. Silvia Einaudi voit dans le choix des chapitres du Livre des Morts des indices de l'élaboration de la célèbre version saïte. Elle a également identifié certaines sources et modèles anciens qui ont inspiré le prêtrelecteur: la tombe d'Itibi (Assiout) pour le texte d'accueil et le temple d'Hatchepsout pour le décor de la salle II. Bernard Mathieu a mis en évidence la fine connaissance qu'avait le prêtre-lecteur du corpus des Textes des Pyramides, mis en scène pour évoquer l'intérieur d'une pyramide à texte. Daniel Werning a souligné les racines abydéniennes de la version du Livre des Cavernes des salles XVII à XIX. Le décor des dernières salles de la tombe (XIX à XXII) puise ses racines dans des compositions thématiques de la XIX dynastie mais recomposées de manière originale, selon C. Traunecker. Enfin, ce dernier a identifié des éléments thématiques propres à Padiamenopé dans le décor des pyramides royales de Kourrou et Nouri au Soudan, ce qui laisse entrevoir une influence du prêtre-lecteur auprès des derniers souverains et souveraines napatéens.

3 À personnage exceptionnel, une tombe tout aussi exceptionnelle, conçue comme une sorte de vade-mecum de la civilisation pharaonique. Mais l'immensité de la tombe et sa réputation en ont fait un monument très visité dès le XVIII ${ }^{\mathrm{e}}$ siècle, période où les torches enflammées étaient un moyen d'éclairage courant. De plus, elle a été utilisée comme lieu d'habitation et d'étable, voire de refuge pour les paysans révoltés contre les taxes. Si on ajoute les colonies de chauves-souris et les pillards de reliefs, on comprend mieux l'état actuel déplorable d'un monument unique dans l'architecture funéraire égyptienne.

4 La réputation d'insalubrité de la tombe de Padiamenopé fait partie d'une longue tradition remontant à l'aube de l'égyptologie. Au début des années 1960, dans ses cours 
à Strasbourg, Jean Leclant ne manquait pas de rapporter l'anecdote des masques en demi-oranges, remplacés toutes les demi-heures, confectionnés par Duemichen pour supporter l'atmosphère méphitique de la tombe de Padiamenopé et stoïquement en copier les textes. Pour nous, alors étudiants, c'était l'image d'une égyptologie rêvée, héroïque et aventureuse; mais en décembre 2005, lorsque nous avons abattu la porte scellée donnant accès aux salles profondes de la tombe, la réalité fétide a inondé nos narines. Une des personnes présentes a souffert pendant plusieurs semaines de troubles olfactifs. Courageusement, mais munis de masques filtrants, nous avons, pendant de nombreuses campagnes, œuvré dans la tombe à l'atmosphère viciée.

Peut-être avions nous sous-estimé la sensibilité de certains d'entre nous aux miasmes de cette tombe. En novembre 2014, C. Traunecker est tombé malade sur place avec des symptômes atypiques et durant l'été 2015 se déclarait une maladie rarissime. Le traitement et les complications en particulier pulmonaires se sont poursuivis sur plus de deux années (embolies, lobectomie, etc.). Sa pathologie avait-elle pour origine l'environnement délétère de la tombe ? Ceci n'a pu être prouvé à ce jour, mais il a tenu à avertir ses collaborateurs des risques potentiels.

6 En 2016, Christine Gallois, présidente du fonds Khéops pour l'archéologie, en collaboration avec Sophie Duberson, restauratrice du département des Antiquités égyptiennes du musée du Louvre, ont consulté divers spécialistes et organisé une première mission d'étude pluridisciplinaire de la tombe sur le plan de sa conservation, de sa mise en valeur mais aussi sur celui de la santé des chercheurs et restaurateurs. Le mécénat financier alloué par une entreprise française (société FORSK) permet depuis 2017 l'organisation de ce programme. Ainsi diverses institutions sont partenaires ${ }^{1}$ de cette étude préalable nécessaire à la compréhension des conditions spécifiques et des mécanismes de dégradation de la tombe de Padiamenopé. Ce programme réunit des restaurateurs et des spécialistes de la préservation du patrimoine qui apportent leurs compétences scientifiques en bio-altération des biens culturels, analyse de la qualité de l'air des environnements intérieurs et examen des phénomènes de dégradation des matériaux liés notamment aux sels solubles. La mise en commun des observations et des résultats permet d'établir un constat de la tombe et de proposer des axes d'amélioration pour rendre le monument plus sain et retrouver la lisibilité des décors peints, en considérant tous les éléments qui font de cette tombe un espace aussi complexe que particulier.

7 À l'instar de ce qui se pratique pour les diagnostics d'espaces clos archéologiques, comme les grottes ornées auxquelles s'apparente la tombe par bien des aspects, les investigations menées dans la TT 33 s'articulent autour de quatre problématiques :

1) Obtenir des données pour décrire et caractériser l'environnement de la tombe : étude climatique et examen des mouvements d'air internes par la mesure des différences de pressions entre les salles.

2) Vérifier l'activité fongique active potentielle et détecter la présence de microorganismes, bactéries et moisissures, en suspension dans l'air (bioaérosols) ou déposés sur les surfaces.

3) Mener une étude sur l'atmosphère de la tombe pour caractériser les composés organiques volatils (COV) présents dans l'air. L'air à l'intérieur de la tombe est en effet, d'après le ressenti des visiteurs, particulièrement irritant et pollué, ce qui impose l'utilisation de protection comme des masques respiratoires. Il était donc urgent 
d'évaluer les risques sanitaires potentiels et d'y remédier pour permettre la poursuite du travail sans, bien entendu, déstabiliser le climat du monument.

4) Enfin, pour comprendre l'origine des altérations observées, il nous fallait caractériser les matériaux en identifiant les composés et produits de dégradation présents par des analyses minéralogiques. Avec 22 salles disposées sur quatre niveaux, la tombe de Padiamenopé est d'une telle superficie qu'il était impossible d'établir un constat précis de chaque paroi dans le cadre d'une mission annuelle. Aussi, notre travail a consisté davantage à relever les faciès d'altérations significatifs de son état matériel afin de mettre en évidence les facteurs d'altérations spécifiques responsables des dégradations.

Les résultats de ces investigations ont permis de caractériser l'air de la tombe et d'envisager des actions d'assainissement pour résoudre tout d'abord la question sanitaire posée pour les épigraphistes, archéologues, restaurateurs et ouvriers travaillant dans le monument. En 2019, un système de ventilation par extraction de l'air vicié a ainsi pu être mis en place, associé à un suivi de son impact et de la qualité de l'air grâce à un ensemble de mesures réalisées avant et après l'extraction (suivi des conditions climatiques, screening couplé à une identification et une quantification des COV, quantification de l'ammoniac atmosphérique et suivi des particules minérales). Enfin, le constat de l'état global de la tombe se précisant, nous avons pu entamer l'examen des moyens de conservation et de restauration à mettre en œuvre.

\section{Travaux réalisés}

\section{1. Épigraphie}

\subsubsection{Textes des passages de portes}

Claude Traunecker

Claude Traunecker a copié les décors de la porte de la salle anépigraphe III-1. Les titres de Padiamenopé sur cette porte sont très originaux. On trouve en particulier le très curieux titre de "prêtre de Iousaâs-du-roi-Horemheb". Karl Richard Lepsius avait observé un cartouche d'Horemheb sur une paroi de la seconde cour (L.D. text, III, p. 245), paroi actuellement inaccessible sans de considérables travaux de dégagements. Cette mention étonnante a alimenté les « historiens » révisionnistes qui veulent faire des Saïtes des contemporains de Toutânkhamon. La découverte du texte complet dans la salle III permet enfin de rendre compte de cette mention apparemment incongrue : Padiamenopé était chargé du culte d'une antique image de la déesse Iousaâs datant du règne de Horemheb. Les titres qu'il porte sur le linteau de cette porte ne se retrouvent pas dans le reste de la tombe.

10 En collaboration avec S. Einaudi, C. Traunecker a trié les fragments des salles II et III. Il a également dressé l'inventaire de boîtes de fragments divers stockés dans la salle I.

\subsubsection{Textes des Pyramides}

Bernard Mathieu

11 Durant sa deuxième participation à la mission, du 27 octobre au 9 novembre 2019, B. Mathieu a pu réaliser les deux objectifs prévus : 1) vérifier la copie des textes de la salle IV, paroi est, effectuée lors de la première mission (novembre 2017) ;2) poursuivre 
et achever la copie des textes de cette même salle. En outre, ont été copiés 3) les courts textes situés dans le passage ( $I V .1 »)$ entre la salle III et la salle IV, ainsi que 4) les premières colonnes de la paroi sud de la salle III.

Tous ces textes relèvent du corpus nommé par la tradition égyptologique «Textes des

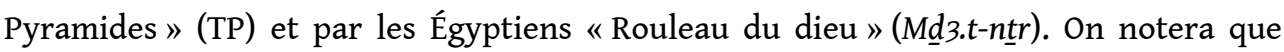
figure précisément parmi les titres de Padiamenopé celui de «scribe du Rouleau du dieu »(š̌ Md $3 . t-n \underline{t} r)$. Il se confirme, à l'examen de différents indices, que les trois salles IV, III et IIIa constituent une réplique des appartements royaux d'une pyramide à textes de l'Ancien Empire, la salle IV correspondant à la chambre funéraire (F), la salle III à l'antichambre (A), et la salle IIIa, anépigraphe, au « serdab » (S).

1) A été vérifiée, dans la salle IV, la copie des textes suivants ${ }^{2}$ : a) le long texte situé en En 1-46, «Interrogatoire du passeur » ou " Formule d'Âqen », dont le prototype figure dans la pyramide d'Aba 587-602 (= Aba/F/Se IV X + 73-88). Ce texte s'est transmis dans les Textes des Sarcophages (TS396 $=$ CT V, 73d-74cc, TS397 $=C T \mathrm{~V}, 75 \mathrm{a}-119 \mathrm{e}$, TS398 $=$ CT V, 120a-160c), ainsi que dans le Livre des Morts (LdM 99); on en connaît actuellement trois autres versions tardives: TT 34 (Montouemhat), TT 27 (Chéchonq), tombe héliopolitaine de Râdjaa (musée du Caire) ; b) la formule d'encensement ou « Texte D «(Es 1-10); c) la séquence TP 72-76 (Es 10-13 + Se 1-3).

2) Les textes occupant les parois Sw - W - Nw ont été copiés. Il s'agit des TP213-219, qui constituent une grande portion de la séquence canonique dite « du grand départ » (TP213-222 + 245-246), séquence qui débute dans toutes les pyramides à textes de l'Ancien Empire sur le mur sud de la chambre funéraire.

Ont également été copiées les cinq colonnes de la paroi Ne (salle IV/Ne 1-5) : il s'agit du TP81, intitulé ici "Formule pour vêtir ce révéré Osiris ritualiste en chef Padiamenopé » (r(3) n(y) ḩbsw jm3H(w) Wsjr hrr(y)-hbb(.t) hrr(y)-tp P(3)-d(w)-Jmn-Jp.t pn).

3) Ont été copiés les courts textes occupant les parois ouest et est du passage («IV.1») entre la salle III et la salle IV. Les textes de la paroi est sont particulièrement intéressants. Le premier (col. 1-8), identifié comme «TS151»(CT II, 255-259; puis LdM 67), doit être plutôt conçu comme une variante du TP ${ }^{\mathrm{N}} 625$ A. Le second (col. 8-12), identifié actuellement comme «TS 625 » (CT VI, 242a-j), fait probablement partie lui aussi du corpus des TP.

16 4) Les parois sud et nord de la salle sont gravées de TP. Une copie préliminaire des 18 premières colonnes de la paroi sud (salle III/S 1-18) a été réalisée; il s'agit des TP 245-246 et 317-319.

\subsubsection{Livre des Morts}

Silvia Einaudi

17 Pendant la campagne 2019, S. Einaudi a poursuivi la copie des formules du Livre des Morts, en achevant d'abord le travail commencé en 2017 dans la salle VI (LdM 147) et ensuite en se consacrant aux textes inscrits dans la salle VII et dans le couloir de passage VIII. Dans ces deux dernières pièces, les parois sont très endommagées et les inscriptions peu lisibles. Néanmoins, l'analyse de ce qui reste a permis d'avoir une idée générale du décor : la salle VII comportait le texte final du LdM 146 (ouest), ainsi que la formule LdM 145 (ouest, sud, est), avec Padiamenopé en adoration devant les génies gardiens des portes de la Douat, dont le nombre reste difficile à déterminer. Quant au couloir VIII, ses parois sont décorées avec les LdM 125a (ouest) et 125c (est) : c'est-à- 
dire le début et la fin de la formule sur la pesée du cœur, dont le reste (LdM 125b ou " confession négative » et la grande vignette) figure dans la salle IX.

Avec C. Traunecker, S. Einaudi s'est aussi occupé du tri, de l'analyse préliminaire et du stockage des fragments décorés, qui étaient déposés sur le sol de la salle II. Certains de ces fragments se sont avérés être des éléments du décor des parois nord et sud de la salle (porteurs d'offrandes) ${ }^{3}$. D'ailleurs, un petit bloc a pu trouver son emplacement d'origine : il représente une partie de la perruque de la figure de Padiamenopé, qui orne la paroi nord du passage entre la salle II et la salle III (fig. 1).

En parallèle des fouilles que S. Nannucci a entrepris dans la salle I (secteur sud-est), S. Einaudi a également mené un premier examen des fragments décorés trouvés dans le sol. Plusieurs blocs peints appartiennent au plafond, tandis que d'autres proviennent de l'encadrement de la porte est de la salle ou du pilastre sud-est. En ce qui concerne ce dernier, on a pu ainsi combler la lacune d'une des vignettes du Livre des Morts figurant sur son côté ouest.

20 La grande quantité de fragments provenant des fouilles, nous impose la mise en place d'un projet pluriannuel de tri, d'analyse et d'enregistrement des blocs (avec une base de données créée ad hoc par une spécialiste), qui débutera lors de la prochaine mission, selon un programme conçu par S. Einaudi. Un groupe de quatre doctorantes égyptiennes mènera cette tâche, sous la direction des épigraphistes de la mission et dans le cadre d'un projet de formation en partenariat avec l'Ifao.

Fig. 1. Exemple de fragment déposé sur le sol de la salle Il et intégrant le portrait de Padiamenopé, paroi nord du passage entre les salles II et III (S. Einaudi).

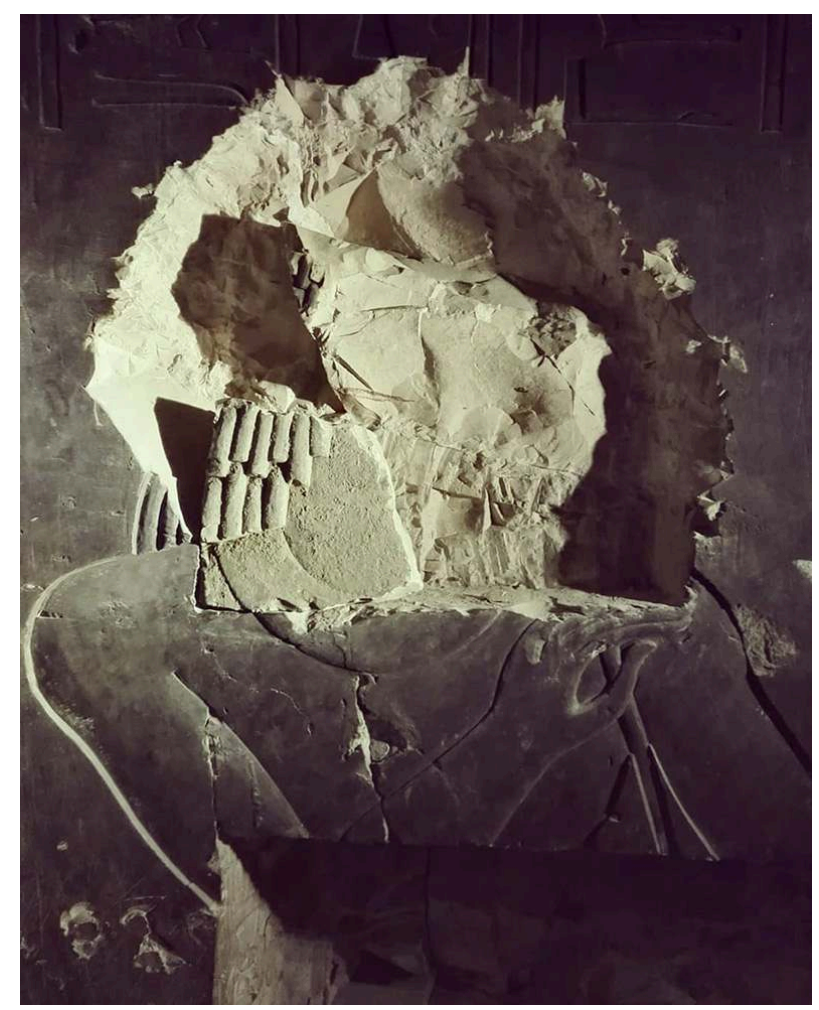

(C) Ifao. 17146_2019_NDMPF_001 


\subsubsection{Amdouat, Livre des Portes et textes associés}

Isabelle Régen

21 Les travaux d'I. Régen se sont concentrés lors de cette campagne sur l'étude du Livre des Portes ${ }^{4}$. Ce corpus, à la diffusion plus confidentielle encore que l'Amdouat, est particulièrement méconnu à l'époque tardive. À ce jour, la version de la tombe de Padiamenopé représente la seule et dernière version complète connue ${ }^{5}$. Cette copie, presque totalement inédite, se déroule sur la paroi sud des salles XII et XIII, occupant une surface d'environ $90 \mathrm{~m}^{2}$. De plus, bien qu'abîmée, la leçon de Padiamenopé présente une originalité, perceptible notamment dans la présence de métadiscours personnalisant au bénéfice du défunt une composition qui fut probablement, à l'origine, un traité cosmographique dont le mort était absent.

22 En confrontant le texte in situ à la couverture photographique et photogrammétrique réalisée par Gaël Pollin et Olivier Onézime (Ifao), I. Régen a pu poursuivre l'élaboration du fac-similé du Livre des Portes sous Adobe Illustrator, en traitant une partie du texte et des scènes de la deuxième heure. En effet, chez Padiamenopé, l'Amdouat et le Livre des Portes se présentent sous une version enrichie : des extraits de textes complémentaires (Livre de la Nuit ; Litanies du Soleil) entrecoupent chacune des heures, offrant ainsi une synthèse originale du parcours nocturne du soleil ${ }^{6}$.

De même, I. Régen a réalisé des vérifications in situ afin de préparer une demande de financement auprès du LabEx Archimède (programme IA-ANR-11-LABX-0032-01), dans l'optique d'achever la production du fac-similé vectoriel du Livre des Portes, par le recours à une prestation externe ${ }^{7}$.

Enfin, elle a documenté (copie et photographie) plus d'une centaine de fragments épigraphiés découverts dans la salle XIII, provenant à la fois du Livre des Portes, du Livre de l'Amdouat, des Litanies du Soleil et du Livre de la Nuit. Les deux fragments au décor le plus remarquable représentent la déesse « Maîtresse de la barque sacrée » (nb.t wj3), provenant selon toute vraisemblance du registre médian de la $6^{\mathrm{e}}$ heure de l'Amdouat ${ }^{8}$, où la divinité figure dans l'équipage de la barque solaire (fig. 2). 
Fig. 2. Fragments représentant la déesse nb.t wj3 [LdA 6]. TT 33, salle XII. Kôm 17 (G. Pollin).

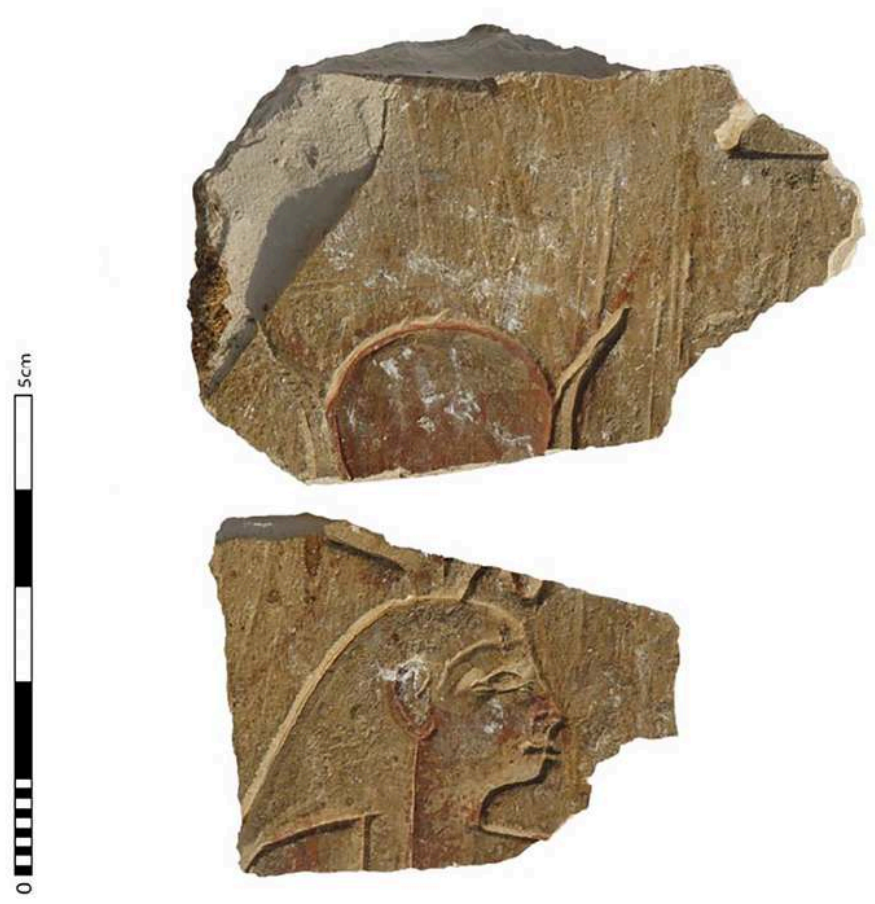

(C) Ifao. 17146_2019_NDMPM_001

\section{Photographie - topographie - photogrammétrie}

\subsection{Photographie}

Gaël pollin

Les prises de vue nécessaires au programme de modélisation de l'ensemble du complexe funéraire de Padiamenopé se sont déroulées cette année du 2 au 13 novembre 2019.

Dans un esprit de continuité et de manière à effectuer la jonction avec la salle I, couverte lors de la mission précédente, l'objectif de cette année fut de réaliser la couverture photographique du porche, de la salle II et des deux passages de porte constitutifs de l'ensemble funéraire.

L'acquisition des images numériques a suivi un protocole contraint par les principes de la photogrammétrie. L'intégralité des parois, plafonds, colonnes et portes d'accès nécessaire à la modélisation a pu être couverte.

En parallèle au travail de terrain, les 1704 clichés composant l'ensemble de la salle I photographiée en 2017 ont été entièrement traités. Ce travail, effectué en collaboration avec le service topographique, a permis de générer des orthoimages d'une qualité suffisante pour servir de support au dessin et permettre une approche philologique des textes. 


\subsection{Topographie - photogrammétrie}

Olivier Onézime

29 $(3,90 \times 2,60 \mathrm{~m})$. Il est situé approximativement dans l'espace entre le pilastre et l'emplacement du premier pilier à gauche de la porte reliant le porche à la salle I et le mur sud (fig. 3). Sa position a été choisie pour vérifier l'épaisseur du dépôt archéologique au-dessus du sol rocheux de la salle à l'endroit où son épaisseur semble être la plus importante. La fouille a révélé une séquence de couches caractérisée par d'abondants restes de paille et d'excréments des différents animaux (particulièrement ânes, bovins, dromadaires et moutons), entrecoupées par des restes de sols de fumier compactés. Aucune couche n'a mis en évidence d'éléments structurels in situ. Au moins deux petits foyers et de légères traces de cendres ont été découverts en relation avec l'un des sols. Deux fosses modernes ont été identifiées près de l'angle sud-est de la salle et près du mur sud.

De nombreux blocs provenant des murs et du plafond effondrés ont été trouvés dans toutes ces couches. Une grande partie des blocs, provenant à la fois des murs d'enceinte et du porche, conserve les restes d'inscriptions et de décorations à relief et peint. La plupart des blocs ne présentent aucune trace de noircissement de la surface. Les débris devaient atteindre au moins $1,70 \mathrm{~m}$ au-dessus du sol rocheux à un moment donné, comme le montre la marque séparant la partie inférieure des murs de la partie supérieure noircie. Il est évident que la partie de cette salle a été utilisée à plusieurs reprises comme étable ou écurie et elle a certainement été vidée jusqu'à la surface rocheuse à un moment donné. Aucune trace de couches contemporaines à l'utilisation de la tombe ou à sa réutilisation dans l'Antiquité n'a été identifiée. 

de nivellement successives liées aux diverses utilisations de cette partie de la tombe effectuées dans le passé (entre autres, étable ou écurie et réserve d'antiquités du SCA). La découverte de restes de momies et de nombreux ossements humains, en plus de la présence de nombreux ouchebtis, suggère une réutilisation de la tombe pour des enterrements secondaires. Il convient de noter qu'au moins une partie des ouchebtis, probablement attribuable à la Troisième Période intermédiaire ou plus ancienne, n'est certainement pas associée à cette réutilisation de la tombe.

La foulle a atteint le sol rocheux de la salle à $50 \mathrm{~cm}$ sous la surface actuelle. La mise en évidence du sol rocheux a permis de déterminer la hauteur du plafond de la salle I à 3,50 m. Le sol rocheux exposé est bien conservé et presque parfaitement horizontal. Il ne présente aucune trace de dallage au-dessus de la surface rocheuse. Son niveau est environ $10 \mathrm{~cm}$ plus bas que le seuil de la porte qui relie le porche et la salle I, ce qui suggère l'existence d'une petite marche à l'entrée de la salle. Sur la base du niveau du sol actuel, on pourrait supposer une disposition similaire pour la porte entre la salle I et la salle II. Les hauteurs décroissantes des plafonds des portes entre le porche et la salle I, entre cette dernière et la salle II et enfin entre la salle II et la salle III, suggèrent l'hypothèse d'une succession de salles légèrement descendantes. Compte tenu de l'irrégularité du sol rocheux que l'on peut observer dans d'autres parties de la tombe, une confirmation définitive ne viendra qu'après la fouille de ces salles.

Fig. 3. Sondage réalisé en novembre 2019 dans une partie de la salle I (S. Nannucci).

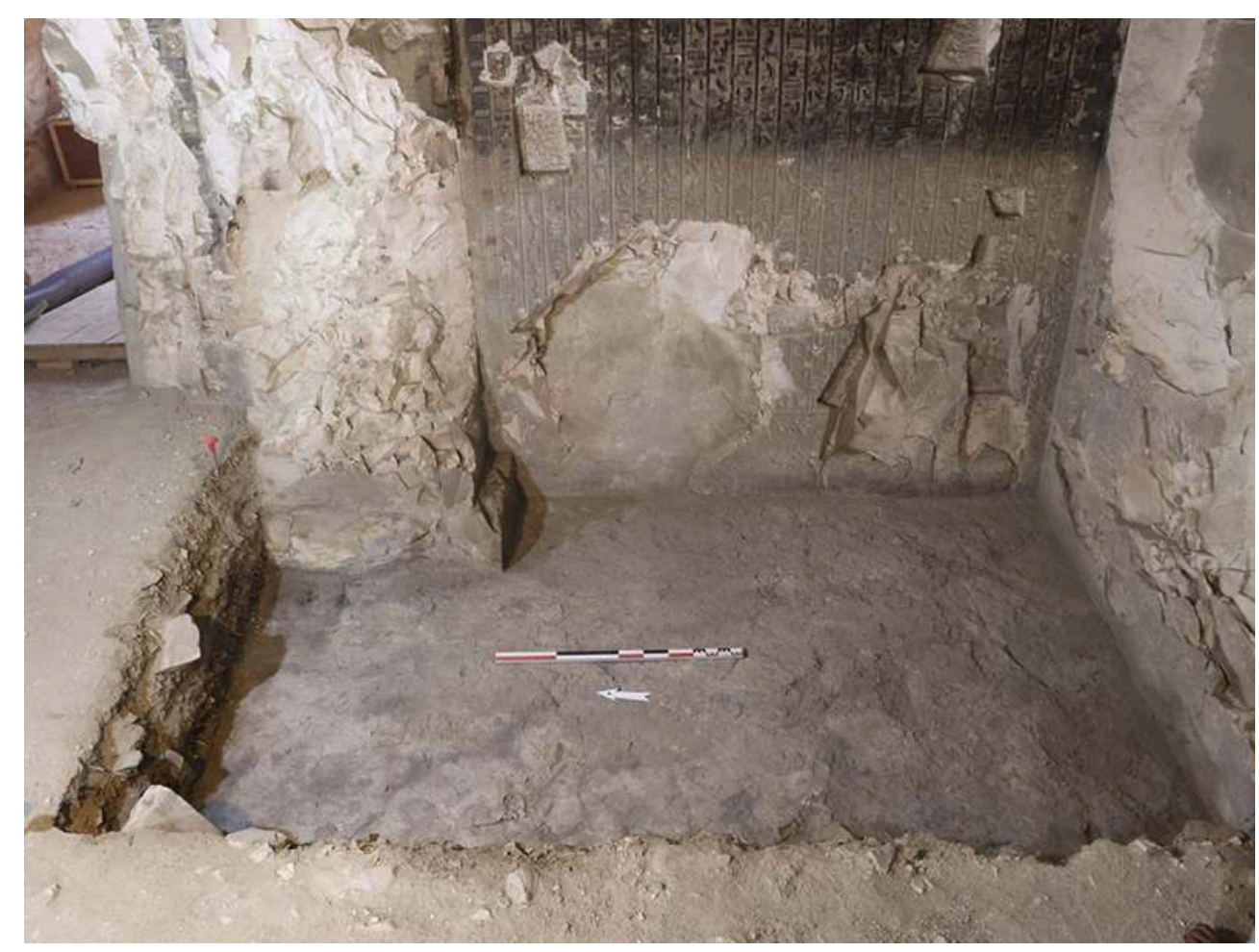

() Ifao. 17146_2019_NDMPF_002

Bulletin archéologique des Écoles françaises à l'étranger , Égypte 


\section{Assainissement et conservation}

Faisl Bousta, François Boyer, Philippe Bromblet, Sophie Duberson, Joëlle Le Roux, Anne Liégey, Stéphane Moularat, Stéphanie Touron

\subsection{Description de l'environnement de la tombe}

L'examen des conditions environnementales a été mené par la prise de mesures climatiques ponctuelles et instantanées (température et humidité relative) et la mise en place, dès 2017, d'un suivi permettant de dresser un profil climatique complet de la tombe grâce à la pose d'enregistreurs thermo-hygrométriques .

Cette étude réalisée par Joëlle Le Roux et François Boyer a montré que le monument présente une inertie tout au long de l'année, l'extérieur n'ayant que peu d'impact sur l'intérieur. C'est un environnement confiné, avec des mouvements d'air infimes vers l'extérieur comme le montre la mesure des différences de pression entre les salles. La tombe présente un climat constant (une température autour de $29^{\circ} \mathrm{C}$ pour une humidité relative de $35 \%$ ) mais avec une distinction importante pour la salle du caveau (salle XXII) située à 20 mètres au-dessous du niveau du sol. Ce dernier constitue à lui seul un espace particulier : à température constante, son HR est plus élevée (65\%) et son poids d'eau dans l'air est nettement plus haut que dans les autres salles, ce qui pourrait laisser supposer l'existence d'une source d'humidité à cet endroit.

Ces données seront complétées à chaque mission. Elles sont essentielles pour assurer une traçabilité dans le temps, évaluer l'impact de l'ouverture et des travaux réalisés dans le monument et éventuellement les corriger si des zones de risque climatique pouvant déstabiliser le monument sont atteintes.

\section{2. Évaluation de la pollution microbiologique}

Cette étude a été réalisée en 2017 pour trois raisons. Tout d'abord, il semblait possible que des micro-organismes se soient développés sur les surfaces décorées ${ }^{10}$. Ensuite, il nous fallait comprendre si un potentiel risque toxique lié à une activité fongique dans l'air existait. Ce travail devait aussi nous permettre de déterminer si un risque de contamination biologique était à redouter en cas de changement de conditions environnementales dû à notre intervention dans la tombe.

La détection de la présence de moisissures et de bactéries et l'identification de leur activité potentielle ont été menées par Faisl Bousta et Stéphane Moularat ; l'analyse du matériel biologique collecté a été réalisée par Dalia Meligny, directrice du Environmental pollution research center, Ministry of Antiquities au Caire.

\subsubsection{Identification des moisissures et bactéries présentes dans l'air et sur les surfaces}

À partir de prélèvements réalisés dans l'air et sur les surfaces solides ${ }^{11}$ puis de l'analyse de leur fraction cultivable en laboratoire, la présence dans la tombe de moisissures et de bactéries a pu être révélée et leur identification réalisée en 2017. Il s'est avéré que la tombe présentait 4 genres fongiques ${ }^{12}$ et 2 genres bactériens ${ }^{13}$ en suspension dans l'air (fig. 4). 
44 prélèvements ont mis en évidence la présence de spores de trois genres fongiques ${ }^{14}$, bien qu'aucun développement fongique actif n'ait été constaté. Cette présence semblait toutefois anodine car elle se caractérise par des taux très bas et par une diversité biologique, bactérienne et fongique, relativement faible. Rappelons que tous les milieux clos comportent des spores en suspension dans l'air ou sur les surfaces.

Fig. 4. Prélèvement de particules biologiques dans l'air de la tombe (F. Bousta).

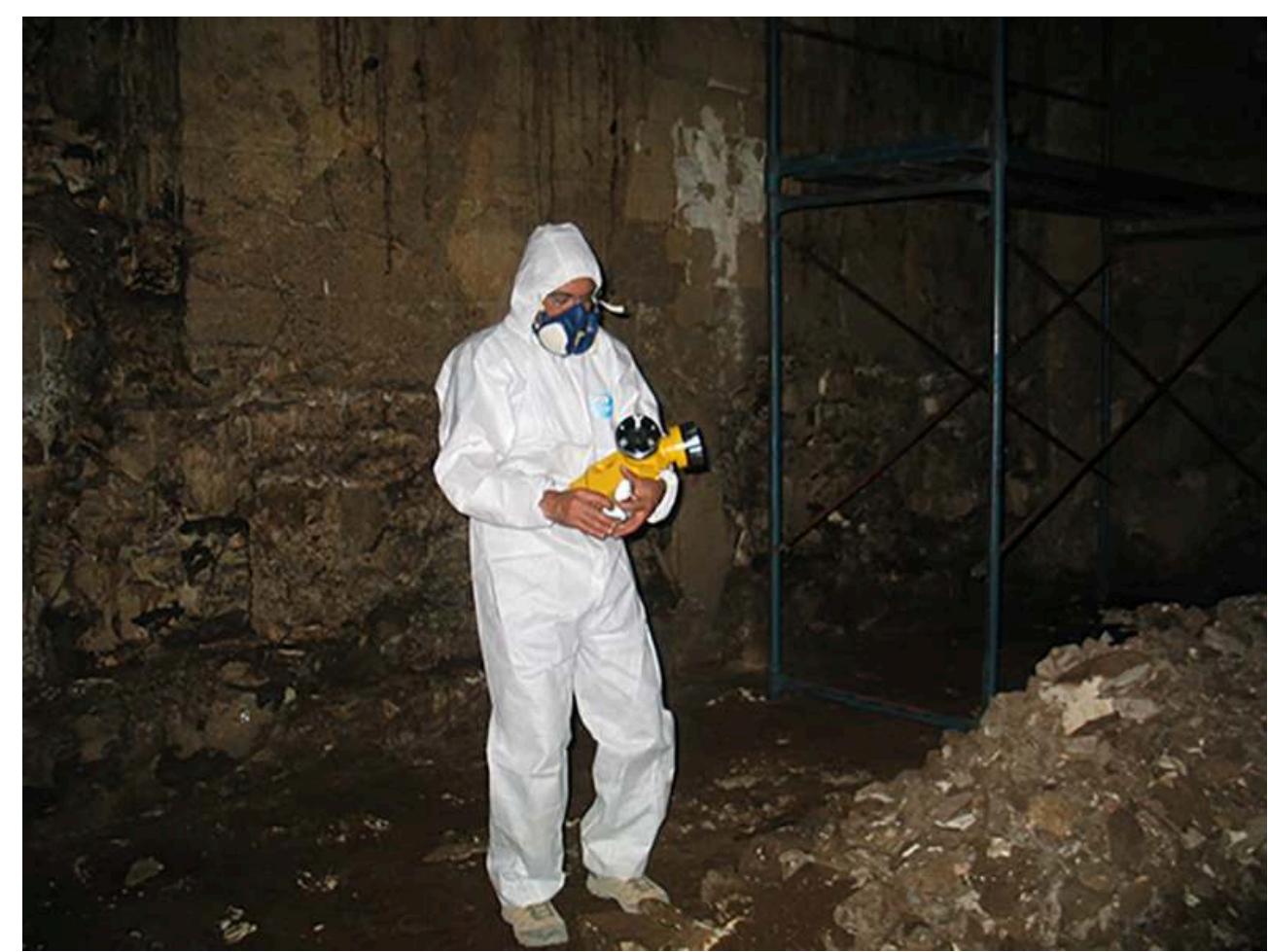

(C) Ifao. 17146_2019_NDMPF_003

\subsubsection{Détection de l'activité métabolique dans les conditions environnementales existantes en novembre 2017}

Les mesures réalisées in situ par F. Bousta par dosage d'ATP ${ }^{15}$ ont montré, en revanche, l'absence d'activité métabolique sur les surfaces indiquant l'absence de biofilm viable. Ainsi, malgré la présence de quelques micro-organismes dans le monument, il n'y a pas de développement fongique actif sur les supports pouvant expliquer un noircissement des parois. De même, le risque de toxicité d'origine fongique a été écarté à partir de prélèvements de poussières ${ }^{16}$ et de la détection de mycotoxines aéroportées, excluant une origine fongique des symptômes ressentis. L'activité biologique pouvait être qualifiée de très faible, ce qui n'excluait pas pour autant la nécessité d'une surveillance de la croissance des espèces puisque le risque d'un développement biologique lors d'un changement d'environnement demeure. Cependant, en l'absence de contamination fongique active (ICF négatif) et de mycotoxines dans l'air, nous avons conclu que l'odeur n'était pas d'origine biologique mais plutôt chimique (notamment composés organiques volatils). 


\subsubsection{Détermination de la qualité chimique de l'air} chimique significative dans toute la tombe. Elle se caractérisait par un nombre important de COV (atmosphère multi polluée). Parmi eux, des composés pouvaient être instables et avoir un impact sanitaire et des effets expliquant l'odeur aigre très désagréable ainsi que le ressenti d'irritation des yeux. On notait en particulier la présence de terpènes, d'acide acétique, de pyridine, d'alpha-pinène et de limonène pouvant être responsables de l'odeur. En revanche, l'ammoniac $\mathrm{NH}_{3}$ que nous pensions reconnaître est en définitive peu présent ${ }^{17}$. Là aussi, une distinction apparaissait entre le caveau et le reste de la tombe, ce dernier présentant une atmosphère spécifique avec une concentration beaucoup plus élevée même si elle restait similaire aux autres salles d'un point de vue qualitatif. L'étude montrait que si aucun des COV présents ne dépassait individuellement les valeurs seuils réglementaires, ils étaient en revanche très nombreux. L'effet de mélange de ces composés n'est pas connu, mais suggère indéniablement une nocivité par inhalation, contact cutané et ingestion, d'autant plus qu'on ignore leur évolution et leur mode de recombinaison en produits secondaires. Ainsi, ces multicomposés formaient ensemble un cocktail improbable se concentrant au fur et à mesure que l'on s'enfonce dans le monument, et notamment dans le caveau, que l'analyse statistique des composés ${ }^{18}$ désignait clairement comme étant un espace particulier sur lequel nous devions concentrer notre attention.

\subsubsection{Origine des COV?}

Bien que ce soit une question essentielle, l'explication de l'origine de la pollution reste pour le moment encore hypothétique. Il semble peu probable que l'odeur et les COV présents dans l'air soient encore issus des déjections des chauves-souris ayant colonisé la tombe jusque dans les années 1950 ou de la décomposition de matériaux divers évacués depuis. En effet, les composés résiduels observés sont anciens, secs et minéralisées et, à l'heure actuelle, ils ne relarguent plus beaucoup de composés. L'atmosphère spécifique constatée à l'intérieur de la tombe pourrait-elle alors s'expliquer par une source de pollution chimique interne, finie ou non, avec de forts soupçons sur le caveau? Cette hypothèse reste possible mais de façon plus vraisemblable, nous pensons que l'origine de la pollution pourrait tout simplement être liée à une concentration des polluants dans la tombe qui n'arrive pas à s'évacuer du fait de l'absence de renouvellement d'air. Cette observation nous a amenés à suggérer l'existence d'une poche de gaz bloquée en fin de galerie dans le caveau en raison probablement de la configuration en $U$ de son accès (effet siphon) (fig. 5). 
Fig. 5. Plan de la tombe présentant la concentration de COVT $\left(\mu \mathrm{g} / \mathrm{m}^{3}\right)$ avec les différentes zones identifiées en 2017 (S. Moularat).

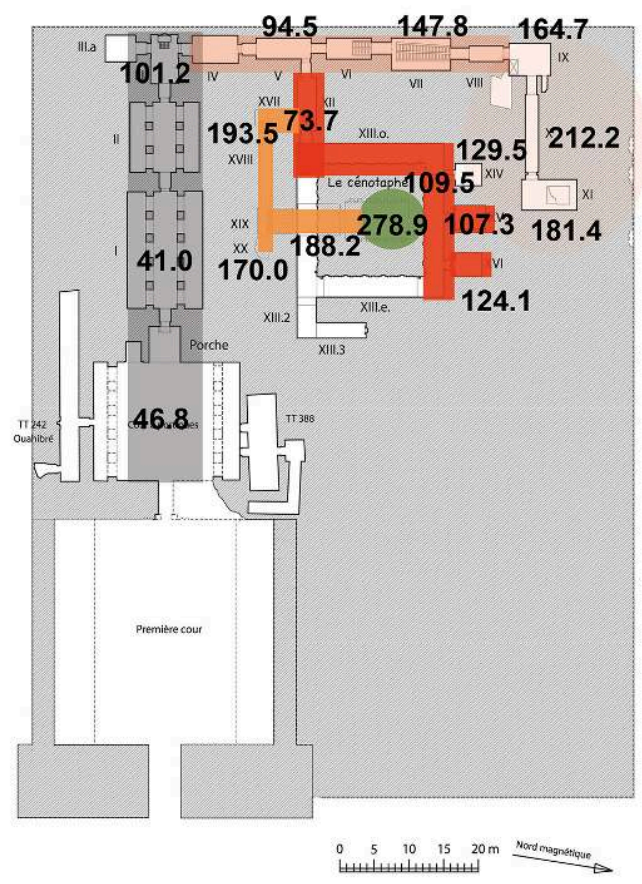

\section{(c) Ifao. 17146_2019_NDMCN_001}

À partir de ce constat, la seule stratégie possible permettant de poursuivre le travail dans la tombe a été de préconiser la mise en place rapide d'un système de ventilation par extraction. Cela semblait être par ailleurs un bon moyen de nous renseigner sur la présence éventuelle d'une source si la pollution se renouvelait après extraction. Ainsi, nous nous sommes concentrés sur le dimensionnement d'un système efficace d'extraction d'air et sur l'évaluation des conséquences possibles de sa mise en place dans le monument.

\subsection{Stratégie de traitement de l'air de la tombe}

\subsubsection{Comment extraire sans déstabiliser l'environnement du monument ?}

L'assainissement de l'air de la tombe a été engagé en octobre 2019 afin d'améliorer la qualité de l'air et de diminuer les risques sanitaires. L'élaboration du système d'extraction d'air a été faite par J. Le Roux et F. Boyer qui ont assuré également son suivi. L'achat du matériel au Caire, la supervision de l'installation et de la mise en fonctionnement du système ont été réalisés sur place par Gérard Ménéroud et C. Gallois.

51 Le choix de la stratégie de renouvellement d'air nous a conduits à privilégier une ventilation par extraction de l'air, plutôt que l'introduction d'air neuf ou autre ${ }^{19}$, avec un unique point d'extraction situé dans le caveau. Ce dispositif permettait de positionner le moteur d'extraction, générant chaleur et éventuellement vibrations, à l'extérieur du monument. Le dimensionnement du système a dû être calculér ${ }^{20}$ 
préalablement à partir du volume du caveau ${ }^{21}$. Il comprenait l'installation d'un extracteur d'air à débit variable situé à l'entrée de la tombe ${ }^{22}$ associé à l'installation d'un réseau de gaine souple de $150 \mathrm{~m}$ de long permettant de rejeter l'air vicié à l'extérieur (fig. 6). Le cheminement de la gaine depuis l'extérieur jusqu'au caveau a été anticipé afin d'évaluer la longueur de gaine nécessaire ainsi que les pertes de charges attendues au niveau des coudes et des puits présents sur le parcours. Avec ce système, le renouvellement d'air est induit par l'entrée d'air neuf progressant de salle en salle à la suite d'une dépression créée dans le caveau. Ainsi, l'air de la tombe est progressivement assaini grâce à l'entrée d'air neuf venant de l'extérieur, qui en se mélangeant à l'air des différentes salles se met petit à petit à la température et à l'hygrométrie du monument, sans déstabiliser le climat ni soulever des poussières en suspension.

La ventilation n'a pas fonctionné en continu puisqu'il nous était impossible de laisser le monument ouvert la nuit. Elle a été réalisée pendant toute la durée de la mission (de mi-octobre à mi-novembre 2019) et ceci, de 7 h00 à 13h00, avec un débit d'air réglé au minimum (moins de $1 \mathrm{~m}^{3} /$ seconde).

Fig. 6. Mise en place d'une gaine souple d'extraction d'air dans la tombe TT 33 à l'automne 2019 (C. Gallois).

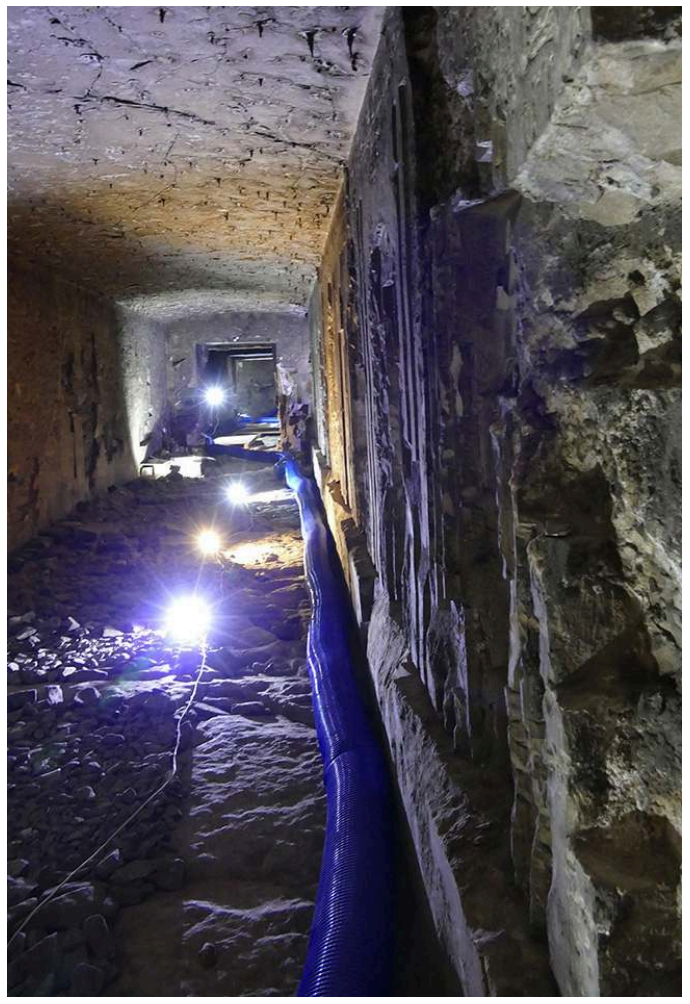

(C) Ifao. 17146_2019_NDMPF_004

\subsection{2. Évaluation de l'efficacité et surveillance de l'impact du système d'extraction d'air}

Différents systèmes de mesures ont été installés pour évaluer les effets de cette ventilation. La surveillance climatique pendant l'extraction a été conduite en continu grâce à un réseau filaire de quatre capteurs de température et d'humidité relative, dont 
une station météo extérieure reliée à un ordinateur. Ce système autorisait un suivi du climat en temps réel et l'enregistrement des valeurs mesurées permettait d'adapter progressivement le débit d'extraction et de mesurer son efficacité. L'analyse des données recueillies a montré que l'impact de l'extraction sur l'environnement climatique a été limité ; en effet, les variations induites par l'apport d'air extérieur ne dépassaient pas les amplitudes tolérées pour la conservation du monument. L'humidité du caveau a baissé pendant l'extraction du fait de l'entrée d'un air sec venant des salles adjacentes mais elle s'élève à nouveau lors de l'arrêt de l'extraction.

Pour évaluer l'éventuel empoussièrement des parois de la tombe lors de l'extraction d'air, des lames de verre recouvertes de ruban adhésif et préalablement pesées, ont été fixées à une hauteur d'environ 1,5 mètre en différents lieux de la tombe. À la fin de l'extraction d'air, les lames ont été récoltées pour être à nouveau pesées, observées et analysées. Les pesées ont montré que seule la lame localisée à l'extérieur de la tombe avait une masse positive qui représentait $233 \mathrm{~g}$ de matière $/ \mathrm{m}^{2}$. Les autres lames contenant très peu de poussières ont une masse négative qui est attribuée au séchage du produit collant sur le ruban adhésif. Leur étude, réalisée par Stéphanie Touron du LRMH, a permis de montrer que la quantité et la taille des particules diminuent à mesure que l'on s'éloigne du caveau. Dans ce dernier, les poussières minérales ont une taille inférieure à $100 \mu \mathrm{m}$. Ensuite, la taille des particules diminue régulièrement de 25 à $10 \mu \mathrm{m}$ lorsque l'on se rapproche de l'extérieur, à l'exception du point de mesure le plus proche de l'extérieur où la taille maximale de poussière est de $30 \mu \mathrm{m}$. Ceci est attribué à une «contamination» de l'air extérieur. L'analyse des particules révèle qu'elles sont constituées de calcite, d'argile et de sels (notamment des sulfates et quelques chlorures).

Ces premiers résultats montrent que la quantité de poussière déplacée lors de l'extraction d'air n'est pas très importante. Néanmoins, il sera nécessaire de les comparer à ceux obtenus lors d'une période sans activité humaine dans la tombe. De nouvelles lames ont été mises en place à la fin de l'extraction d'air; elles seront collectées lors de la prochaine mission et permettront de connaître le taux d'empoussièrement normal de la tombe lorsqu'il n'y a aucune activité humaine.

Avant et après la mise en marche de l'extraction, l'analyse de l'air ambiant a été une nouvelle fois réalisée en mesurant la qualité chimique de l'air grâce à des pompes reliées à deux séries de tubes dotés de réactifs pour l'ammoniac et les COV. La stratégie d'échantillonnage a été établie en fonction des mesures statistiques en composantes principales des salles permettant de cibler trois zones représentatives d'atmosphères se distinguant les unes des autres (salle V, salle XI et salle XXII, c'est-à-dire le caveau). Par ailleurs, des mesures ponctuelles de différentiel de pression et de débit d'air réalisées avant et après extraction nous ont donné des indications sur la vitesse et sur le sens de circulation de l'air entre les salles sur l'ensemble de la tombe.

57 L'analyse des données recueillies démontre l'efficacité du traitement. En effet, non seulement la baisse progressive et régulière des concentrations en COV a pu être constatée, mais on s'aperçoit également que l'ensemble de la tombe est impacté de façon homogène. La ventilation a généré des mouvements d'air dans tout le monument mis en évidence par une augmentation significative des différentiels de pression (dans la plupart des salles autour de $15 \mathrm{~Pa}$ après extraction alors qu'ils n'étaient que de 1 à $2 \mathrm{~Pa}$ avant extraction en 2017). L'extraction a donc atteint toutes les salles sans laisser de zones mortes. 

est particulièrement visible sur les composés qui étaient en forte concentration au départ $\left(>8,5 \mu \mathrm{g} / \mathrm{m}^{3}\right)$. Par exemple, l'alpha pinène qui présentait une concentration très élevée de $698 \mu \mathrm{g} / \mathrm{m}^{3}$ dans le caveau a été réduite à $74 \mu \mathrm{m} / \mathrm{m}^{3}$ après ventilation. On est parvenu avec ce système à abaisser les concentrations moyennes. Ainsi, la tombe semble s'homogénéiser (dans le caveau, la moyenne de la concentration est passée de $40,17 \mu \mathrm{g} / \mathrm{m}^{3}$ à $6,16 \mu \mathrm{g} / \mathrm{m}^{3}$; salle V de $6,49 \mu \mathrm{g} / \mathrm{m}^{3}$ à $4,18 \mu \mathrm{g} / \mathrm{m}^{3}$ et salle $\mathrm{X}$ de $9,05 \mu \mathrm{g} / \mathrm{m}^{3}$ à $\left.6,27 \mu \mathrm{g} / \mathrm{m}^{3}\right)$.

Si on examine ces résultats de façon qualitative, on observe des réactions différentes en fonction du type de COV :

- Les COV totaux (TVOC) ont été réduits de plus de $80 \%$ dans le caveau, plus de $55 \%$ en salle V et plus de $25 \%$ en salle $X$.

- Les COV très volatils (VVOC) ont été réduits de plus de $90 \%$ dans le caveau et stable en salles $\mathrm{V}$ et $\mathrm{X}$.

- Les COV semi-volatils (SVOC) ont été réduits de plus de $72 \%$ dans le caveau mais augmentés de plus de $55 \%$ en salle $V$ et apparus en salle X. En fait, il faut s'attendre à ce que ces COV plus lourds mettent plus de temps à s'évacuer. L'augmentation apparente peut également s'expliquer par la diminution du bruit de fond général du monument (avec la ventilation) rendant identifiable certains COV.

\subsubsection{Qualité de l'air en 2019}

En 2019, avant extraction, 63 composés ont été identifiés (alpha pinède, limonène, acide carboxylique et acide acétique, dont de nombreux composés se trouvant dans les huiles essentielles) parmi lesquels 19 ont des concentrations supérieures à $5 \mu \mathrm{g} / \mathrm{m}^{3}$. Si la plupart sont des composés classiques, comme en 2017, c'est leur nombre et leur combinaison qui font que cette atmosphère est atypique.

61 Curieusement, les analyses révèlent également des variations de composés entre la campagne 2017 et 2019 avant extraction. L'atmosphère semble avoir changé. On remarque de façon non significative que certains sont apparus (3-carene par exemple) tandis que d'autres ont augmenté (composés benzéniques) et que d'autres encore ont disparu (la pyridine n'a pas été retrouvée en 2019). Cela peut s'expliquer parce que les composés étaient présents sur les parois dans une atmosphère saturée avant extraction et ont alors désorbé, ou encore en raison de réactions après un changement d'équilibre de composés entre eux. Plus simplement, il est possible aussi que leurs pics d'analyse cachés par d'autres en concentration plus importante soient apparus avec la diminution des composés majoritaires.

L'extraction a donc été non seulement efficace et douce mais aussi homogène sans que des zones mortes ne soient constatées. Les mesures réalisées chaque matin avant la remise en route du système ont montré qu'il n'y avait pas de remontée significative des concentrations pendant la nuit. Cela semble indiquer que le phénomène attendu de désorption de polluants par les parois est en définitive limité et long, ou encore compensé par l'extraction.

63 L'analyse des données aussi intéressante soit elle pour comprendre la nature des composés, leur impact sur la santé et éventuellement leur origine nous montre surtout que la stratégie de ventilation adoptée produit un effet réel sur l'atmosphère de la tombe qui s'en trouve modifié. Celle du caveau notamment a particulièrement changé. 
Néanmoins, l'assainissement n'est à ce jour pas encore total et il devra être poursuivi. Il semble évident maintenant que le processus nécessitera un certain temps, surtout pour les composés les moins concentrés ou pour les COV semi-volatils. Selon l'évolution des données après une longue période d'inactivité de la ventilation, les mesures de contrôle de l'air à venir nous permettront de confirmer le phénomène supposé de désorption des parois ainsi que la présence d'une source potentielle.

\subsection{Analyse des produits d'altération et conservation du monument}

L'intérieur de la tombe se caractérise par l'empoussièrement important des parois et par leur noircissement et leur souillure due principalement à des projections organiques sur les zones d'accroches des chauves-souris. À ces altérations manifestes et facilement observables s'ajoutent diverses dégradations résultant, entre autres, de l'action des sels solubles sur les matériaux. Cet état, rapidement décrit ici, est en lien avec l'histoire de l'occupation du monument et l'évolution naturelle de la cavité. Il est ancien comme le montrent les témoignages écrits et les photos d'archives ${ }^{23}$.

Cependant, afin d'en comprendre plus précisément l'origine et l'évolution, des analyses des cristallisations salines, concrétions diverses, efflorescences, stalactites et autres produits d'altération présents sur les parois ont été nécessaires (fig. 7). Cette étude (DRX et dosages des sels solubles) a été menée par Philippe Bromblet qui a travaillé avec différents laboratoires du Caire ${ }^{24}$ coordonnés par Ashraf Nagueh, vice-directeur du Center of Research and conservation of Antiquities.

Fig. 7. Analyse des concrétions sur les parois du caveau (S. Duberson).

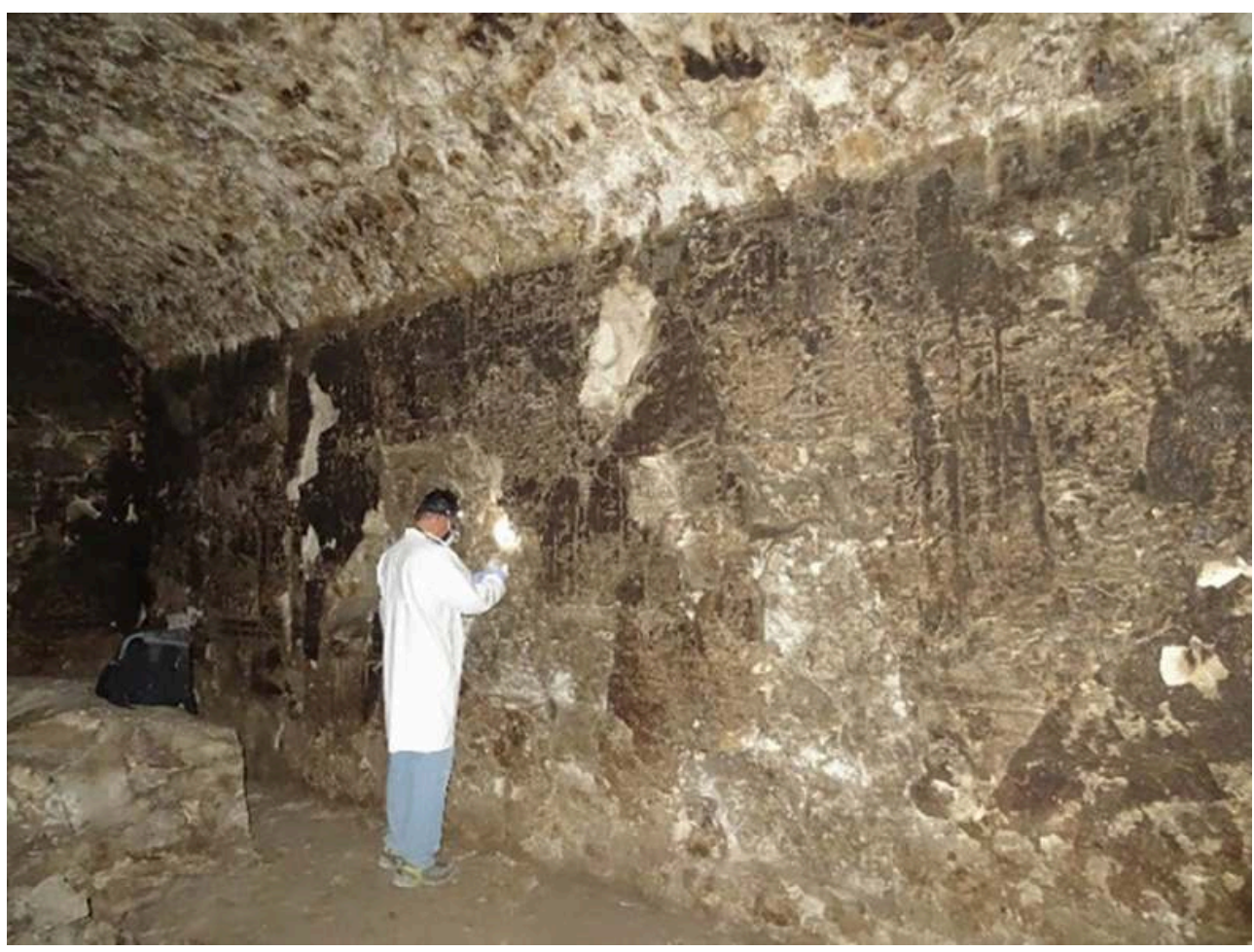

(C) Ifao. 17146_2019_NDMPF_005 


\subsubsection{Composition des croûtes noires}

D'après les résultats que nous avons pu obtenir des laboratoires impliqués, il apparaît que les dépôts noirâtres obscurcissant sont constitués principalement d'un mélange de suies carbonées, d'excréments de chauve-souris en cours de minéralisation (formation de composés azotés types urée et de phosphates) et de sels solubles (il s'agit principalement de halite, chlorure de sodium et de sulfate de calcium) qui lient les microcristaux des particules. Les sels solubles semblent avoir cristallisé à partir de solutions qui s'infiltrent très lentement à travers la roche calcaire. L'épaisseur, la composition et l'aspect de ces couches noires varient en fonction des zones et des surfaces (fig. 8). Elles se présentent en pellicule fine, en couches poudreuses granuleuses ou encore croûtes épaisses compactes d'aspect vitreux et luisant assez particulier. Les coupes montrent clairement que ces composés ont une grande interaction avec le substrat et qu'ils ont migré dans la porosité de la roche en la colorant (fig. 9). Dans cette configuration, leur retrait ne pourra s'opérer qu'à la surface des matériaux; il est nécessaire d'admettre qu'on ne pourra donc pas aboutir partout à des nettoyages restituant un état de surface proche de celui d'origine.

Dès lors, dans le cas particulier de ce monument, le nettoyage des parois n'est plus une option. Si on considère la quantité importante de sels solubles dans la composition des couches noires, leur retrait devient une problématique de dessalement qui devra, dans la mesure du possible, être effectué sans eau afin de ne pas remobiliser les sels en surface. Qui plus est, non seulement les tests de nettoyage réalisés ont montré que la dissolution de ces composés par voie chimique est peu efficace ${ }^{25}$ mais de plus, il nous semble illogique d'employer des produits chimiques dans une atmosphère déjà très polluée. L'ensemble de ces éléments nous incite donc à nous orienter plutôt vers des nettoyages par voie mécanique de type micro-abrasion. 
Fig. 8. Couche noire contenant des sels solubles (P. Bromblet).

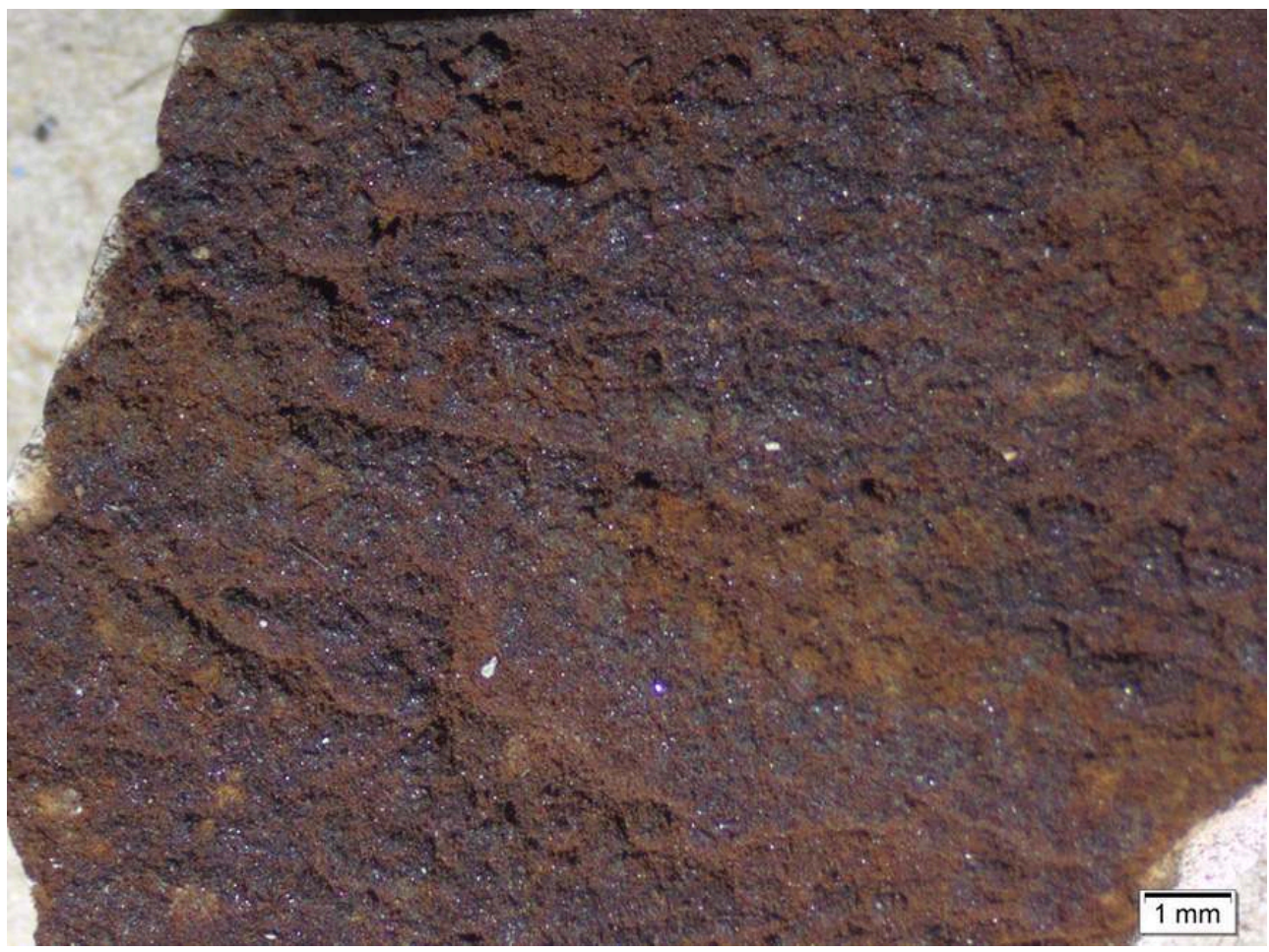

(c) Ifao. 17146_2019_NDMPM_002

Fig. 9. Interaction de la couche noire avec la pierre (P. Bromblet).

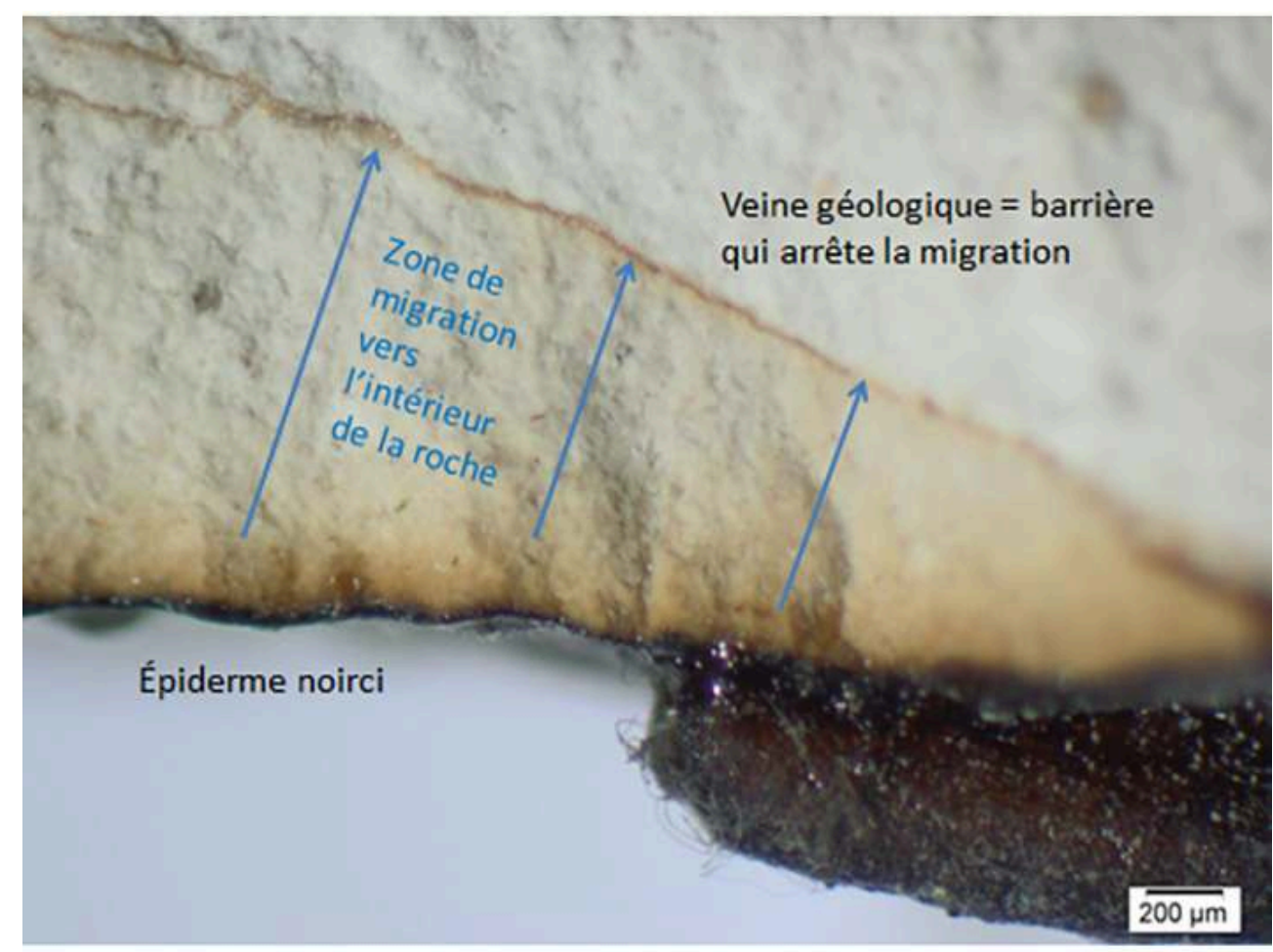

(C) Ifao. 17146_2019_NDMPM_003 


\subsubsection{Altérations structurelles des matériaux}

l'ensemble de la tombe est difficile à définir étant donné sa superficie et la variété des surfaces à traiter, qui naturellement ne présentent pas partout les mêmes problématiques. L'élaboration d'un protocole complet demande du temps et surtout, doit nécessairement être l'aboutissement logique des études préliminaires menées sur les matériaux et sur le contexte de la tombe. La demande des responsables du monument et des autorités concerne généralement, et de façon bien légitime, la mise en valeur de la tombe par le nettoyage très attendu des parois peintes (restauration). Cette logique n'est pas toujours celle des restaurateurs qui donnent plus volontiers la priorité au traitement des altérations potentiellement évolutives pouvant aboutir à des pertes d'informations immédiates (conservation), jugé plus urgent et moins facultatif. Toutefois, pour répondre au mieux aux contraintes du site, parallèlement aux études scientifiques, la mise en œuvre de tests de retrait de la couche noire a été engagée dès 2017 afin d'évaluer l'efficacité, l'innocuité et la faisabilité des procédés classiques de restauration dans un tel espace, pollué et fragilisé.

71 Il est tout à fait remarquable, dans le cas particulier de cette tombe, de constater comment l'étude scientifique et les essais pratiques de nettoyage ont convergé progressivement vers une conclusion analogue car comme nous l'avons dit, l'ensemble de ces travaux nous permet aujourd'hui d'écarter raisonnablement toutes méthodes chimiques de nettoyage, aqueuses ou non, pour privilégier des méthodes mécaniques.

Du point de vue du restaurateur, les tests ${ }^{26}$ de nettoyage par micro-sablage de précision ${ }^{27}$ menés en 2019 sur des parties fracturées de paroi ont donné une orientation de traitement prometteuse et satisfaisante du point de vue de l'efficacité et de la préservation des surfaces (fig. 10). Pour les parties présentant des restes de polychromie, l'emploi de cet outil doit encore être optimisé aux abords de zones présentant des couleurs très fragiles (celles à granulométrie importante moins liées comme le bleu égyptien) lors de notre prochaine campagne. 
Du point de vue scientifique, le nettoyage est présenté comme étant une intervention qui participe à la dépollution de la tombe en permettant l'évacuation des composés organiques semi-volatils. Les COVS sont des composés ayant la propriété d'être volatils quand ils sont dans l'air mais de devenir des particules lorsqu'ils s'adsorbent sur les parois. Quand nous avons compris que le film noir semblant s'intensifier sur les surfaces peintes n'était pas biologique, l'hypothèse d'une saturation des surfaces en COVS a été proposée. On suppose désormais que ces particules lourdes, quand elles sont collées aux parois, ne seront pas évacuées par la ventilation mais qu'une action mécanique sera nécessaire pour les éliminer et ceci dans une atmosphère assainie afin d'éviter le risque de re-déposition après déplacement des équilibres thermodynamiques.

Fig. 10. Test de nettoyage par micro-abrasion sur la surface peinte d'un pilier, salle III (S. Duberson).

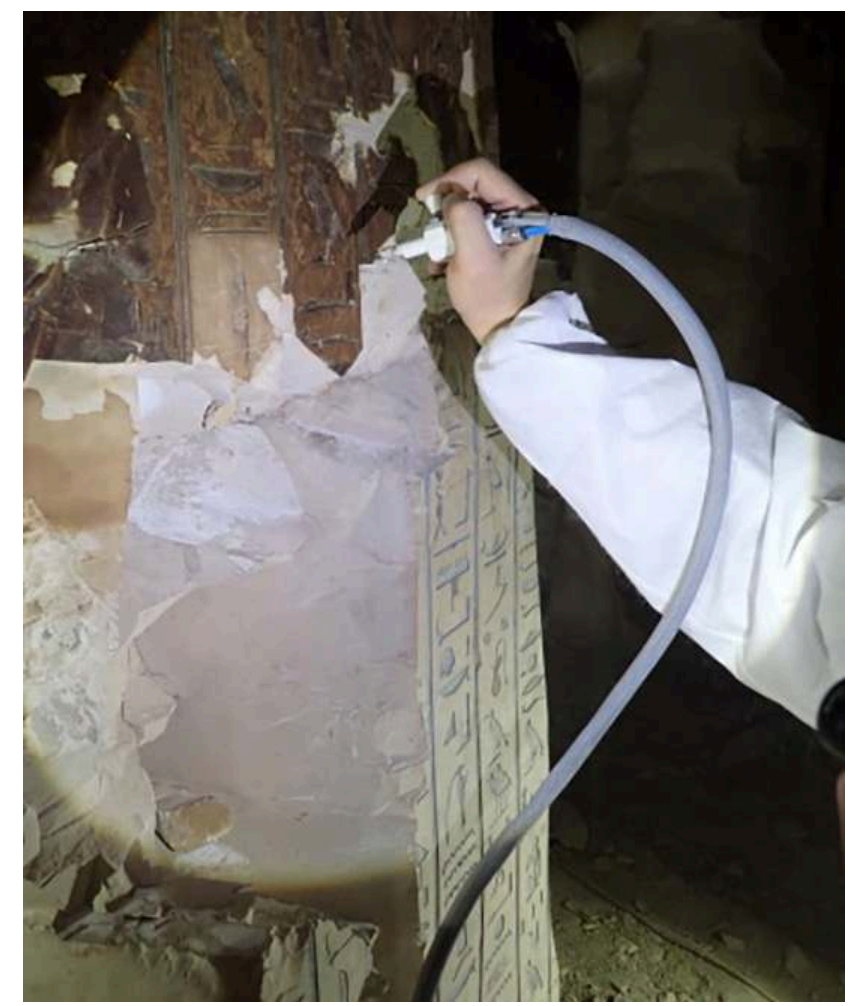

(C) Ifao. 17146_2019_NDMPF_006

La difficulté que nous devons à présent résoudre consiste à trouver des solutions de mise en œuvre des techniques de nettoyage pour évacuer en même temps, et les abrasifs et les particules semi-volatiles remises en suspension dans l'air, par une aspiration puissante, immédiate et proche de la zone en traitement pour que l'équilibre se refasse à nouveau. Les prochaines campagnes permettront d'affiner le protocole de restauration et d'envisager la formation d'une équipe de restaurateur, le chantier de restauration proprement dit ne pouvant débuter qu'au terme de la fouille archéologique des sols et de l'évacuation des fragments tombés. 


\section{Communications scientifiques}

75 - Silvia Einaudi, «Les textes funéraires dans les tombes monumentales de l'Assassif: une vue d'ensemble ", table ronde des 13-14 février 2019, "Les textes funéraires à la Troisième Période intermédiaire. État de la question » organisée par Florence Albert et Giuseppina Lenzo à l'Ifao, Le Caire, 14/02/2019.

76 - Isabelle Régen, «La transmission du Livre de l'Amdouat de la TPI à la Basse Époque », table ronde des 13-14 février 2019, "Les textes funéraires à la Troisième Période intermédiaire. État de la question « organisée par Florence Albert et Giuseppina Lenzo à l'Ifao, Le Caire, 13/02/2019.

77 - Claude Traunecker, «De l'organisation de l'Éternité de Padiamenopé: décor et fonctions des salles ultimes de la TT 33 (sources et antécédents) », table ronde des 13-14 février 2019, « Les textes funéraires à la Troisième Période intermédiaire. État de la question » organisée par Florence Albert et Giuseppina Lenzo à l'Ifao, Le Caire.

\section{Publications}

\subsection{Monographies}

WERNING 2019

Daniel A. Werning, Das Höhlenbuch im Grab des Petamenophis (TT 33). Szenen, Texte, Wandtafeln. Berlin, Edition Topoi, 2019.

79 EINAUDI (sous presse 1)

Silvia Einaudi, La rhétorique des tombes monumentales tardives. Une vue d'ensemble de leur architecture et des leurs programmes décoratifs, déposée.

\subsection{Articles}

EINAUDI (sous presse 2)

Silvia Einaudi, «Le fil rouge qui relie Padiamenopé à Hatchepsout. La formule 607 des Textes des Sarcophages dans la TT 33 », sous presse.

81 EINAUDI (sous presse 3)

Silvia Einaudi, «Le programme décoratif des tombes tardives de l'Assassif : reflet de croyances, pratiques cultuelles et savoir ", sous presse.

ENGELMANN-VON CARNAP 2018

Barbara Engelmann-von Carnap, «Zum Mundöffnungsritual im Grab des Padiamenope (TT 33) ", BIFAO 118, 2018, p. 127-141.

83 RÉGEN 2020

Isabelle Régen, « Menkhéperrê A, Padiamenopé (TT 33) et Nectanébo II. La transmission du Livre de l'Amdouat, de la Troisième Période intermédiaire à la Basse Époque ", BIFAO 120, 2020 (manuscrit accepté).

84 RÉGEN (sous presse 1)

Isabelle Régen, «Le rwd de Padiamenopé (TT 33). Architecture, archaïsme et paysage rituel dans l'Assassif », sous presse. 
Isabelle Régen, "Le fonds Alexandre Piankoff », in Jérôme Gonzalez (éd.), Mémoires d'archives. Cinquantième anniversaire de la bibliothèque d'égyptologie de Montpellier, sous presse.

Claude Traunecker, "Tombe de Padiamenopé (TT 33)» in Laurent Coulon, Mélanie Cressent (éd.), Archéologie françaises en Égypte, Le Caire, Ifao, 2019, p. 216-221.

87 TRAUNECKER (sous presse 1)

Claude Traunecker, "Padiamenopé (TT 33) et les nécropoles royales soudanaises", dans Actes du $14^{e}$ Congrès international des Études nubiennes, musée du Louvre 2018, sous presse.

88 TRAUNECKER (sous presse 2)

Claude Traunecker, « Tombeaux de l'élite et tombeaux de rois. De la montagne thébaine à la quatrième cataracte » in Pharaon des Deux Terres. L'épopée africaine des rois de Napata, catalogue de l'exposition Louvre, section 3, chapitre 2, sous presse.

WERNING 2018

Daniel A. Werning, «The Book of Caverns in Theban Tomb 33: Late Period Reception Process and Individual Adaptation », BIFAO 118, 2018, p. 525-554.

\section{Valorisation de la recherche (conférences grand public, articles de presse, documentaires, etc.)}

90 Carnet de la mission épigraphique française dans la tombe de Padiamenopé (Plateforme OpenEdition): https://tombett33.hypotheses.org/ (création et administration : Isabelle Régen).

\section{Articles de presse}

\subsection{Presse régionale}

91 Encart dans Midi Libre, édition du mercredi 3 juillet 2019, page 3 (tombe TT 33): «L'équipe de chercheurs engagée sur des chantiers»: https://www.midilibre.fr/ 2019/07/03/montpellier-plongee-dans-les-tresors-de-la-bibliotheque-degyptologie, 8292276.php

\subsection{Conférences grand public}

92 - Claude Traunecker, Silvia Einaudi, La missione archaeologica nella tombe di Padiamenope a Luxor, Saluzzo (FAI, Delegazione di Saluzzo), 30 mai 2019.

- Isabelle Régen, «The labyrinthic tomb of the priest Padiamenope (TT 33) ». VII Semana de Egiptologia do Museu Nacional, Rio de Janeiro (Brésil), 04/09/2019. 


\section{NOTES}

1. Au département des Antiquités égyptiennes du musée du Louvre, qui coordonne le projet, se sont associés le service de la conservation préventive à la direction de la recherche et des collections du musée du Louvre, le laboratoire de recherches des monuments historiques à Champs sur Marne (LRMH), le centre interdisciplinaire de conservation et restauration du patrimoine à Marseille (CICRP), le centre de recherche et de restauration des musées de France (C2RMF) et le centre scientifique et technique du bâtiment (CSTB). Nous remercions les directions de ces institutions d'avoir accepté de participer à cette étude.

2. Rappel : dans la salle IV, le système de numérotation de Dümichen doit être modifié, dans la mesure où ce dernier a numéroté les colonnes de textes sans tenir compte des changements de paroi. Les TP se répartissent en deux séries, l'une à l'ouest (parois SwW-Nw) et l'autre à l'est (parois Es, Se, En et $\mathrm{Ne}$ ). La seconde série présente une subdivision, de part et d'autre d'un axe d'où se développent deux séquences, l'une qui va du nord au sud (Es 1-13 + Se 1-3) et l'autre du sud au nord (En 1-46 + Ne 1-5).

3. S. Einaudi, «Le fil rouge qui relie Padiamenopé à Hatchepsout. La formule 607 des Textes des Sarcophages dans la TT 33 », sous presse.

4. L'étude de ce corpus est incluse dans le programme d'enseignement 2019-2020 de sa charge de conférences à l'EPHE, sur la chaire de Laurent Coulon (« Religion de l'Égypte ancienne ») : https://www.ephe.fr/formations/conferences/religion-de-l-egypteancienne-cours-ccf; http://www.archeo.ens.fr/La-litterature-funeraire-egyptiennearcheologie-du-rituel-et-transmission.html.

5. Les seules autres versions complètes connues du Livre des Portes datent du Nouvel Empire (sarcophage de Séthy I ${ }^{\mathrm{er}}$; tombe de Séthy I ${ }^{\mathrm{er}}$; Osiréion d'Abydos).

6. Du reste, le tableau final du Livre des Portes est enrichi d'une scène du Livre de Nout présentant une forme spécifique aux tombes tardives de la nécropole de l'Assassif.

7. La demande de financement ayant été obtenue, le contrat du dessinateur débutera le $1^{\text {er }}$ mai 2020.

8. La déesse apparait à chacune des heures de l'Amdouat, à l'exception de la $7^{\mathrm{e}}$ heure. La localisation des fragments a été déduite de la recherche de lacunes pertinentes, l'état de conservation exceptionnel de la surface décorée, des conditions uniquement rencontrées sur la paroi nord de la salle XII, mais également du lieu de découverte (au nord du Cénotaphe).

9. Température et humidité relative exprimée en \% (désigne le degré de saturation de l'air ou, autrement dit, le rapport entre l'humidité absolue que porte l'air par rapport à l'humidité absolue maximale que cet air pourrait porter).

10. Il semblait aux épigraphistes que certaines zones anciennement allégées en dépôts noirs par les restaurateurs du service des antiquités égyptiennes avaient renoirci, ce qui n'a pu être démontré jusqu'à présent.

11. La qualification des bioaérosols se fait grâce à des prélèvements d'air réalisés in situ avec un impacteur d'air (système SAS) qui capte les particules biologiques, champignons et bactéries en suspension dans l'air, lesquelles sont ensuite impactées sur un milieu cultivable. Les prélèvements permettant de qualifier la biomasse présente 
sur les surfaces se font à l'écouvillon frotté sur les parois. Les microorganismes poussant sur des milieux synthétiques sont mis en incubation pour pouvoir ensuite être identifiés et quantifiés par comptage des germes (exprimés en unités formant colonies (UCF) par $\mathrm{m}^{3}$ d'air).

12. $52 \%$ de la population de spores fongiques en suspension dans l'air était composée de plusieurs espèces d'aspergillus et $14 \%$ de trois espèces de genre Penicillium; les $2 \%$ restant étant composé de Chaetomimm globosum.

13. L'analyse des aérosols biologiques révèle des taux de particules fongiques, moisissures et bactéries, en suspension dans l'air allant de $160 \mathrm{UFC} / \mathrm{m}^{3}$ d'air à $380 \mathrm{UFC} /$ $\mathrm{m}^{3}$.

14. Caldosporium, Aspergillus, Penicillium et Chaetomium.

15. Détection d'adénosine triphosphate (ATP), molécule présente dans tous les organismes vivants. Le taux détecté indique la viabilité des microorganismes présents sur les surfaces et permet de déterminer in situ la présence d'une éventuelle activité biologique.

16. La caractérisation de l'activité fongique active a été déterminée grâce au calcul de l'indice de contamination fongique (ICF) réalisé in situ à partir de 17 points de prélèvements, dans le cadre de l'étude de la pollution COV. Elle s'appuie sur la recherche des composés organiques volatils d'origine microbienne (COVm) émis lors des premières heures de l'activité fongique d'un milieu, ce qui constitue une empreinte biochimique détectable. Cette mesure a été faite en 2017 grâce à 17 points de prélèvements repartis sur l'ensemble de la tombe.

17. Les analyses 2019 ont montré que sa concentration avant extraction était de 1,24 E-05 ppm, c'est-à-dire 30000 fois inférieure au seuil d'odeur qui est de 0,6 à 53 ppm.

18. ACP ou analyse en composantes principales est une méthode d'analyse statistique permettant de décrire les ambiances chimiques détectées dans la tombe.

19. La dépollution in situ avec une filtration continue avait été écartée pour des raisons de sécurité (moteur électrique émettant de la chaleur et d'éventuelles étincelles au sein d'une atmosphère chargée en polluants), de difficulté d'acheminement du matériel dans le caveau et de coûts (l'utilisation de filtres aux charbons actifs pouvant se révéler très coûteux si la pollution est une source active).

20. L'aide de deux climaticiens du musée du Louvre a été précieuse pour dimensionner le système ; merci donc à Christian Ritter et Vincent Samson pour leur contribution.

21. Soit un volume de $250 \mathrm{~m}^{3}$, ce qui a déterminé le choix du moteur d'extraction puisque sa puissance a été évaluée en fonction du renouvellement d'air souhaité.

22. Débit $500 \mathrm{~m}^{3} / \mathrm{h}$ permettant un renouvellement d'air de 0,5 à 2 volume/heure.

23. La tombe est décrite dans la littérature depuis le milieu du XviII ${ }^{\mathrm{e}}$ siècle, ce qui nous indique qu'elle était ouverte et visitable et cela depuis très longtemps probablement. Ces textes nous livrent surtout des informations pittoresques sur la présence des chauves-souris et l'odeur pestilentielle à l'intérieur de la cavité, à défaut d'être réellement utiles pour la compréhension de l'état actuel de la tombe. Ainsi, le noircissement complet de la cavité n'est pas expliqué autrement que par des hypothèses de réutilisation du lieu ou de feux volontairement allumés pour chasser les volatiles, forcement répétitifs et ceci sur une très longue période. Cela n'explique pas 
pour autant l'étendue générale des dégâts qu'on peut donc malgré tout qualifier de très anciens.

24. Chemical Administration, Ministry of Trade and Industry, Center of research and conservation of Antiquities du CSA.

25. Les tests réalisés ont d'abord mis en œuvre des méthodes chimiques de nettoyage classiquement utilisées lors de la restauration des tombes thébaines. Leur utilisation s'est révélée sans action (niveau de nettoyage très faible) et peu contrôlable (compresses de papier ou gels de nettoyage mettant en œuvre différents produits actifs : solvants, bicarbonate d'ammonium ou EDTA à des concentrations correspondant aux normes acceptables). Les pourcentages des concentrations ne pouvant être augmentés du fait de la sensibilité des couches picturales, nous avons très vite renoncé à ce type de traitement.

26. Micro sableuse CTS1, abrasif : oxyde d'alumine de granulométrie 220 mesh.

27. Technique de nettoyage usuelle dans le domaine de la conservation et de la restauration des biens culturels consistant à projeter sur la zone à nettoyer un jet d'air comprimé sec sous faible pression (de 0,5 à 3 bars) véhiculant des particules de poudre abrasive inerte de granulométrie calibrée qui font éclater la salissure. Le phénomène de retrait des dépôts à nettoyer dépend de nombreux paramètres : la nature du matériau à éliminer, les propriétés de la poudre (granulométrie, dureté et forme des particules), la pression du jet d'air qui détermine la pression de l'impact, le diamètre de la buse, le débit d'abrasif, la distance et l'angle entre la buse et la surface traitée. L'emploi d'un appareil de micro-sablage est un acte technique qui nécessite une formation car selon la dextérité de l'utilisateur (constance du geste) et sa capacité à effectuer les réglages requis, cette méthode de nettoyage peut créer des rugosités sur des surfaces fragiles ou aboutir à des nettoyages irréguliers.

\section{INDEX}

Année de l'opération : 2019

sujets https://ark.frantiq.fr/ark:/26678/pcrttgndozev0k, https://ark.frantiq.fr/ark:/26678/ pcrtMw5xXEAy1F, https://ark.frantiq.fr/ark:/26678/crtqPFI1HPTo2, https://ark.frantiq.fr/ark:/ 26678/crtnWIFXB4hFo, https://ark.frantiq.fr/ark:/26678/pcrtbQbkP4KBeG, https:// ark.frantiq.fr/ark:/26678/crtxd88uhXtEM, https://ark.frantiq.fr/ark:/26678/pcrtJrInD7k8G1, https://ark.frantiq.fr/ark:/26678/pcrt69L0JofMDy

Thèmes : IFAO

\section{AUTEURS}

\section{CLAUDE TRAUNECKER}

Égyptologue, université de Strasbourg, CNRS, UMR 7044 Archéologie et histoire ancienne : Méditerranée-Europe ArcHiMedE 
BERNARD MATHIEU

Égyptologue, université Paul-Valéry Montpellier 3, CNRS, UMR 5140

SILVIA EINAUDI

Égyptologue, CNRS-PSL-EPHE, UMR 8546 Archéologie et philologie d'Orient et d'Occident AOrOc, université de Strasbourg, CNRS, UMR 7044

\section{ISABELLE RÉGEN}

Égyptologue, université Paul-Valéry Montpellier 3, CNRS, UMR 5140 Archéologie des Sociétés Méditerranéennes

\section{GAËL POLLIN}

Photographe, Ifao

\section{OLIVIER ONÉZIME}

Topographe-photogrammètre, Ifao

\section{SIMONE NANNUCCI}

Archéologue, université de Strasbourg, UMR 7044

FAISL BOUSTA

Responsable du pôle Microbiologie, LRMH

\section{FRANÇOIS BOYER}

Pôle conservation préventive, $\mathrm{C} 2 \mathrm{RMF}$

\section{PHILIPPE BROMBLET}

Géologue, CICRP

SOPHIE DUBERSON

Restauratrice, département des Antiquités égyptiennes, musée du Louvre

JOËLLE LE ROUX

Restauratrice, service de la conservation préventive à la direction de la recherche et des collections du musée du Louvre

\section{ANNE LIÉGEY}

Restauratrice indépendante

STÉPHANE MOULARAT

Spécialiste des polluants en milieu fermé, Centre Scientifique et Technique du bâtiment - CSTB STÉPHANIE TOURON

Responsable du pôle Grottes Ornées, LRMH 